\title{
Caries dental en escolares de 6 a 12 años de edad en Navolato, Sinaloa, México: experiencia, prevalencia, gravedad y necesidades de tratamiento
}

\author{
Juan José Villalobos-Rodelo ${ }^{1}$, Carlo Eduardo Medina-Solís ${ }^{2,3}$, Nelly Molina-Frechero ${ }^{4}$, \\ Ana Alicia Vallejos-Sánchez ${ }^{5}$, América Patricia Pontigo-Loyola ${ }^{2}$, José Luis Espinoza-Beltrán ${ }^{6}$ \\ ${ }^{1}$ Facultad de Odontología, Universidad Autónoma de Sinaloa, Culiacán, Sinaloa, México. \\ 2 Área Académica de Odontología, Instituto de Ciencias de la Salud, Universidad Autónoma del Estado de \\ Hidalgo, Pachuca, Hidalgo, México. \\ ${ }^{3}$ Centro de Investigación en Sistemas de Salud, Instituto Nacional de Salud Pública, Cuernavaca, Morelos, \\ México. \\ ${ }^{4}$ Departamento de Atención a la Salud, División de Ciencias Biológicas, Universidad Autónoma Metropolitana \\ Xochimilco, México D.F., México. \\ ${ }^{5}$ Facultad de Odontología, Universidad Autónoma de Campeche, Campeche, México. \\ ${ }^{6}$ Subdirección de Salud Bucal, Servicio de Salud de Sinaloa, Culiacán, Sinaloa, México.
}

Introducción. La caries dental es una de las enfermedades más prevalentes en los niños. Objetivo. Determinar la experiencia, prevalencia y gravedad de caries en la dentición temporal y permanente; establecer igualmente el índice de caries significativa ( $\mathrm{SiC}$, por sus siglas en inglés), en escolares de Navolato, Sinaloa, México, así como sus necesidades de tratamiento. Materiales y métodos. Se realizó un estudio transversal en 3.048 niños de 6 a 12 años de edad. Los sujetos fueron examinados clínicamente por tres examinadores (índice $\kappa>0,85$ ), de acuerdo a los lineamientos establecidos por la Organización Mundial de la Salud (OMS). Para la detección de caries se empleó el criterio de la OMS y la lesión d1 de Pitts.

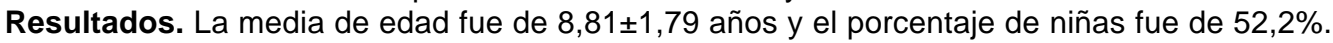
En la dentición temporal, el ceod (Promedio de dientes cariados, extraídos/perdidos y obturados) fue de 4,68 $\pm 3,21$ y la prevalencia de caries $90,2 \%$ (ceod $>3=60,8 \%$ ). El índice significativo de caries fue de 10,52 para los niños de seis años de edad. Las lesiones no cavitadas (d1) representaron 37,1\% del componente "cariados". En la dentición permanente, el índice CPOD (Promedio de dientes permanentes cariados, perdidos y obturados) fue de $3,24 \pm 2,72$ y la prevalencia de caries, $82 \%$ (CPOD $>3=47,8 \%$ ). El índice de caries significativa fue de 10,87 para los niños de 12 años. Las lesiones no cavitadas representaron $63,4 \%$ del componente "cariados". Al menos $81,1 \%$ de los niños necesitó la restauración de una superficie dental y $89,6 \%$, de dos. Las niñas presentaron más caries que los niños en su dentición permanente. Se observó que conforme aumentó la edad se incrementó la experiencia de caries.

Conclusiones. Se observaron altos índices de caries, así como alta prevalencia, gravedad y necesidad de tratamiento. Las lesiones no cavitadas constituyeron el principal porcentaje en la dentición permanente, no así en la dentición temporal.

Palabras clave: salud bucal, epidemiología, encuestas de salud bucal, caries dental, índice CPO, niño, México.

Dental caries in schoolchildren aged 6-12 years in Navolato, Sinaloa, México: experience, prevalence, severity and treatment needs

Introduction. Dental caries is the most prevalent disease in children.

Objective. To determine the caries experience, prevalence, and severity in temporal and permanent dentition, likewise to establish the significant caries index in schoolchildren of Navolato, Sinaloa, Mexico as well as their treatment needs.

Material and methods. A cross sectional study in 3048 schoolchildren aged 6 to 12 years. All subjects were clinically examined by three examiners (kappa>0.85), according to World Health 
Organization (WHO) guidelines. Caries detection criteria used were the WHO's criteria and Pitts' lesion $\mathrm{d}_{1}$.

Results. The mean age was $8.81 \pm 1.79$ years old and $52.2 \%$ were girls. In the temporal dentition; the dmft was $4.68 \pm 3.21$ and caries prevalence of $90.2 \%$ (dmft>3 was $60.8 \%$, and $\mathrm{dmft}>6$ was $27.4 \%$ ). The overall significant caries index was 10.52 for 6 year olds. The noncavitated lesions $\left(\mathrm{d}_{1}\right)$ represented $37.1 \%$ of the "decay" component. In the permanent dentition; the DMFT was $3.24 \pm 2.72$ and caries prevalence was $82.0 \%$ (DMFT>3 was $47.8 \%$, and DMFT>6 was $9.0 \%$ ). The overall significant caries index was 10.87 for 12 year olds. The noncavitated lesions $\left(d_{1}\right)$ represented $63.4 \%$ of the "decay" component. The majority, $89.6 \%$ and $81.1 \%$ of children needed at least one restoration of one and two dental surfaces, respectively. Girls were more affected by caries than boys in permanent dentition, but not in temporal dentition. The caries experience increased with age.

Conclusions. We observed high indices of caries, and high prevalence, severity and treatment needs. Noncavitated lesions were higher in permanent dentition that in primary dentition.

Keywords: oral health, epidemiology, dental health surveys, dental caries, DMF index, child, Mexico.

La caries dental es un proceso que implica un desequilibrio de las interacciones moleculares normales entre la superficie y la subsuperficie del diente y la capa microbiana adyacente. Este desequilibrio se manifiesta en un cierto plazo como desmineralización acumulativa del diente que, si es desenfrenada, tiene el potencial de producir cavitación del esmalte y daño colateral a la dentina y a la pulpa, culminando con la destrucción localizada de los tejidos duros del diente (1). Se considera que la gravedad y el costo social de la caries dental en niños de edad preescolar y escolar son considerables. Diversos autores (2-10) han indicado que es un problema de salud pública debido a su alta prevalencia e incidencia; además se caracteriza por encontrarse concentrada en poblaciones con desventajas sociales en México y el mundo.

Según varios autores (11-14), la prevalencia de caries ha tenido una disminución en países desarrollados. Igualmente, la prevalencia y la gravedad de la caries entre 1970 y 2000 en niños de cinco a seis años y de 11 a 13 años ha mostrado una disminución significativa en el contexto latinoamericano (15). Desde los 70 se ha documentando la caries dental como un problema

Correspondencia:

Carlo Eduardo Medina Solis

Privada de Altillo s/n entre Av. Central y Pedro Moreno, Colonia San José, CP. 24040, Campeche, Campeche, México.

Telefax: 52 (981) 81 10215. cemedinas@yahoo.com

Recibido: 21/09/06; aceptado: 29/03/06 de salud en México (16). En la encuesta efectuada por la Secretaría de Salud en 1980 sobre morbilidad bucal en escolares del Distrito Federal, los datos señalan que los niños de 6 a 14 años de edad presentaron un alto porcentaje de caries (95,5\% en dientes permanentes) (17). Informes posteriores en esa misma década y en los 90 la ubicaban por arriba de 90\% (18-20). En otros estados como Guadalajara y Campeche, sin embargo, se observaban prevalencias mucho menores. Así, en 1998, Mendoza y cols. reportaron prevalencias de 17,9 y $62,5 \%$ para los grupos de edad de 6 y 12 años, respectivamente (7). En 1997 en Campeche, Casanova y cols. reportaron por su parte un promedio de más de tres dientes afectados y una prevalencia de poco más de $80 \%$ en la dentición permanente de los niños de 12 años de edad (6). En ese mismo estado y año, Vallejos y cols. reportan una prevalencia por encima de $70 \%$ y 2,5 dientes afectados en promedio. Mucho más recientemente, después de la fluoruración de la sal de mesa en el Estado de México se reportaron 2,5 dientes cariados, perdidos u obturados en la población de 12 años de edad (21).

En la ciudad de Navolato, Sinaloa, no hay precedentes de estudios sobre salud bucal en niños escolares. Por este motivo, el objetivo del presente estudio fue establecer la experiencia (promedio de dientes ceod y CPOD y superficies ceos y CPOS dentales temporales/permanentes cariados, extraídos/perdidos y obturados), la prevalencia (porcentaje de niños con dientes 
temporales/permanentes cariados, extraídos/ perdidos y obturados, ceod/CPOD > 0) y la gravedad de la caries (ceod/CPOD $\geq 3$ y ceod/ $\mathrm{CPOD} \geq 6$ ) en la dentición temporal y permanente e, igualmente, determinar el índice de caries significativa ( $\mathrm{SiC}$ ) en escolares de Navolato, Sinaloa, así como establecer sus necesidades de tratamiento.

\section{Material y métodos}

\section{Diseño y población de estudio}

Sinaloa se ubica en el noroeste de México, limita al norte con los estados de Sonora y Chihuahua, al este con Durango y Nayarit, al sur con Nayarit y el Océano Pacífico y al oeste con el Golfo de California y Sonora. Su superficie de $58.092 \mathrm{Km}^{2}$ lo ubica en el décimo séptimo lugar con 2,9\% de la superficie total del país (22). El municipio de Navolato se encuentra en el centro del Estado, con una densidad de población de 51 a 100 por $\mathrm{Km}^{2}$. En el 2000, el municipio contaba con una población de 145.396, ocupando el quinto lugar en población entre los 18 municipios del estado, o sea $5,7 \%$ (23).

La realización de este estudio cumplió con las especificaciones de protección a los participantes en estudios y se adhirió a las reglamentaciones éticas y de investigación del Instituto Nacional de Salud Pública en vigor.

Se realizó un estudio transversal en niños de 6 a 12 años de edad asistentes a todas las 18 escuelas primarias de la ciudad de Navolato, Sinaloa. La población blanco constó de 3.547 niños. Para realizar este estudio no se realizó ningún tipo de muestreo. En la primera fase se habló con los representantes del sector salud y educativo de la ciudad, posteriormente se invitó a las madres/tutores de los niños a participar en el estudio. Del total de niños, en una primera fase se obtuvo una tasa de respuesta de $75,4 \%$ ( $n=$ 2.674). En un segundo recordatorio se obtuvo una tasa de respuesta final de $87,0 \%(n=3.086)$. Los niños fueron excluídos del estudio $(n=38)$ por alguna de las siguientes razones:

a) tenían menos de seis años y más de 12 años de edad, b) tuvieran alguna enfermedad que comprometiera la cavidad bucal, c) rehusara practicarse el examen bucal, y d) tuviera aparatologia ortodóntica fija. Por lo que la muestra final fue de $3.048(85,9 \%)$ escolares.

\section{Variables del estudio}

Se calcularon los índices epidemiológicos tradicionales ceod, ceos, CPOD, CPOS y el nuevo índice de caries significativo (SiC) (24) desarrollado a partir de las limitaciones que presenta el CPOD en su distribución sesgada. Este índice hace referencia al tercio de la población que se encuentra más afectada por caries, y es en este tercio que se calcula el índice CPOD. Las variables ceod y CPOD fueron las variables dependientes. Los niños con ceod y $C P O D=0$ se clasificaron como sujetos sin caries. Para el análisis de los datos se utilizó también el índice de cuidados (Care index) que muestra los cuidados retauradores a los que la población ha estado expuesta por medio de la relación (25):

dientes obturados

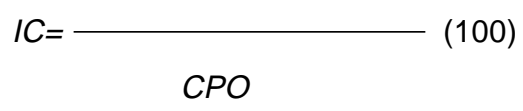

al igual que el índice de necesidades de tratamiento, el cual se calculó con la siguiente fórmula (26):

$$
\text { INT }=\frac{\text { dientes cariados }}{\text { dientes cariados }+ \text { dientes obturados }}(100)
$$

\section{Recolección de los datos}

Antes de pasar a la recolección de los datos, se procedió a realizar un ejercicio de estandarización del criterio para la detección de caries a través de una prueba piloto. Todos los sujetos fueron examinados clínicamente por uno de tres examinadores capacitados y estandarizados (índice $\kappa>0,85$ ) en el criterio de caries de acuerdo a los siguientes lineamientos establecidos por la OMS (27): para la medición de las variables clínicas se utilizó un espejo bucal plano, sonda dental CPI y luz natural (método visual/táctil); se consideró diente presente cuando alguna parte de éste era visible; cuando un diente permanente y un diente primario ocupaban el mismo espacio, se asentó el estado del permanente; si existía duda, el diente se clasificaba como sano. 
En un formato diseñado para el estudio se asentaron los resultados de las variables clínicas. El examen bucal consistió en la detección de la experiencia de caries (caries, obturaciones y extracciones). En este estudio se incluyeron no sólo las lesiones obviamente cavitadas, sino también las lesiones d1 del criterio de Pitts $(28,29)$. Estas lesiones se incluyeron para medir de una manera adecuada el desarrollo longitudinal de la caries dental en esta población.

\section{Análisis de los datos}

Como primer paso se procedió a la captura de la información en una base de datos en el paquete estadístico SPSS 10 y posteriormente se depuraron. A continuación se procedió con el análisis univariado, el cual consistió en el cálculo de medidas de tendencia central y de dispersión para las variables continuas como la edad y los índices de caries, y de porcentajes para las variables categóricas como el sexo.

Igualmente, se estableció la relación entre la presencia de caries en la dentición temporal y permanente con el cálculo de la razón de momios (RM). Al tratarse de una población, no fue necesario calcular el intervalo de confianza. Los procedimientos estadísticos se realizaron en STATA 8.2.

\section{Resultados}

El promedio de edad de los niños incluidos en este estudio fue de $8,81 \pm 1,79$, y el porcentaje de mujeres fue de $52,2 \%$. Se evaluaron los dientes permanentes de 3.018 niños y la dentición temporal de 2.623. Treinta sujetos presentaron sólo dentición temporal, 425 sólo dentición permanente y 2.593 tenían dentición mixta al momento del estudio. El número de dientes examinados fue de 43.670 dientes permanentes y 29.104 temporales. El promedio de dientes presentes en boca por sujeto fue de $14,33 \pm 6,98$ dientes permanentes $y$ $9,55 \pm 5,82$ dientes temporales.

El promedio del índice ceod fue de 4,68 $\pm 3,21$, mientras que el CPOD fue de $3,24 \pm 2,72$. A los 12 años el CPOD fue de 6,49 $\pm 3,85$. El promedio de superficies cariadas, perdidas y obturadas en la dentición temporal fue de $10,08 \pm 9,17$ y de $7,08 \pm$ 6,49 para la dentición permanente. En la figura 1 se presenta la distribución del promedio de dientes cariados, perdidos y obturados temporales por grupo de edad, mientras que la figura 2 presenta los resultados para la dentición permanente. El componente "caries" de los índices ceod y CPOD contribuyó con el mayor porcentaje (83,3 y 95,4\%, respectivamente). En un análisis realizado entre las escuelas incluidas en el estudio observamos diferencias entre ellas, con prevalencias de caries en la dentición permanente que estuvieron entre 60,0 y $95,8 \%$ y entre 79,2 y $100 \%$ en la dentición temporal.

Las figuras 3 y 4 muestran los resultados de la prevalencia y gravedad de las caries en la

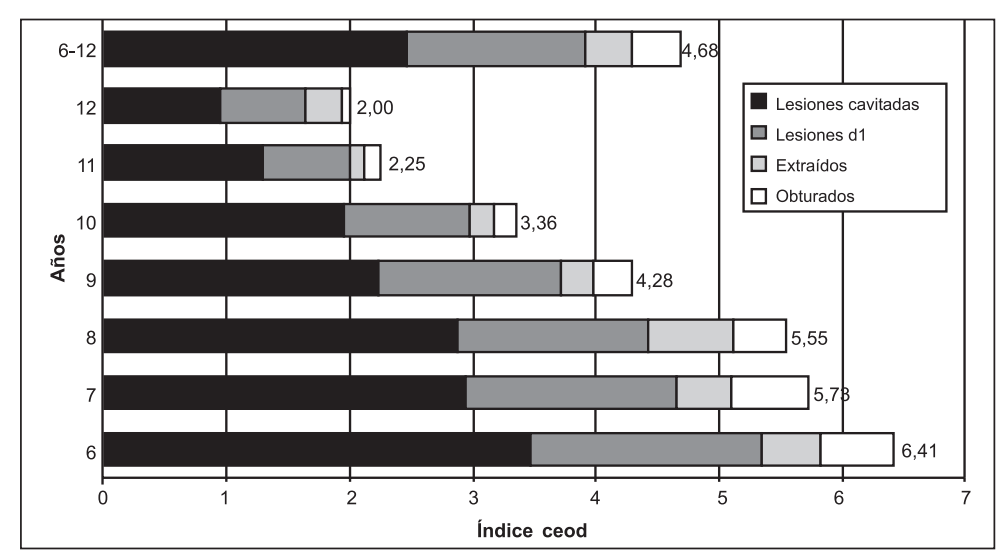

Figura 1. Distribución de los dientes temporales cariados, dientes extraídos/indicados para extracción, dientes obturados e índice ceod en niños de 6 a12 años de edad de Navolato, Sinaloa, México, 2004. 


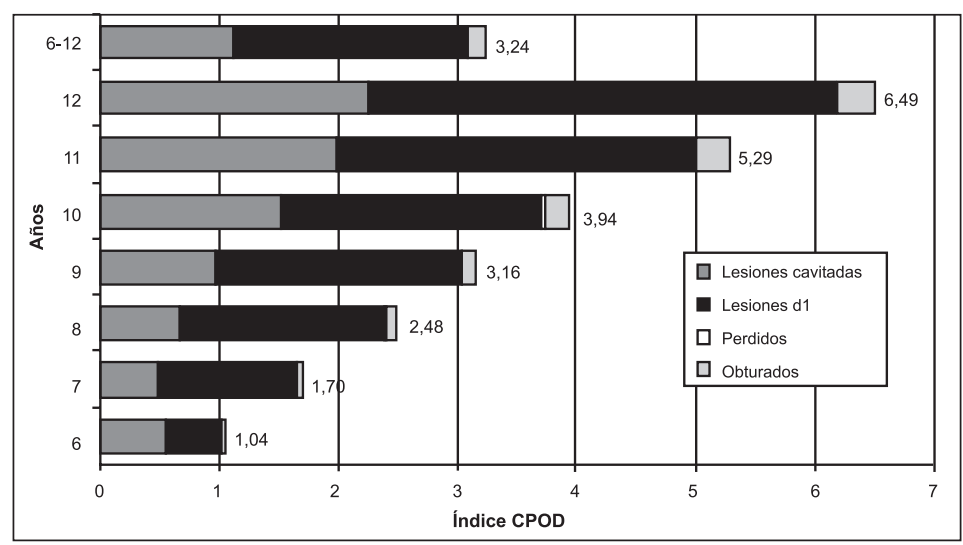

Figura 2. Distribución de dientes permanentes cariados, dientes perdidos, dientes obturados e índice CPOD en niños de 6 a12 años de edad de Navolato, Sinaloa, México, 2004.

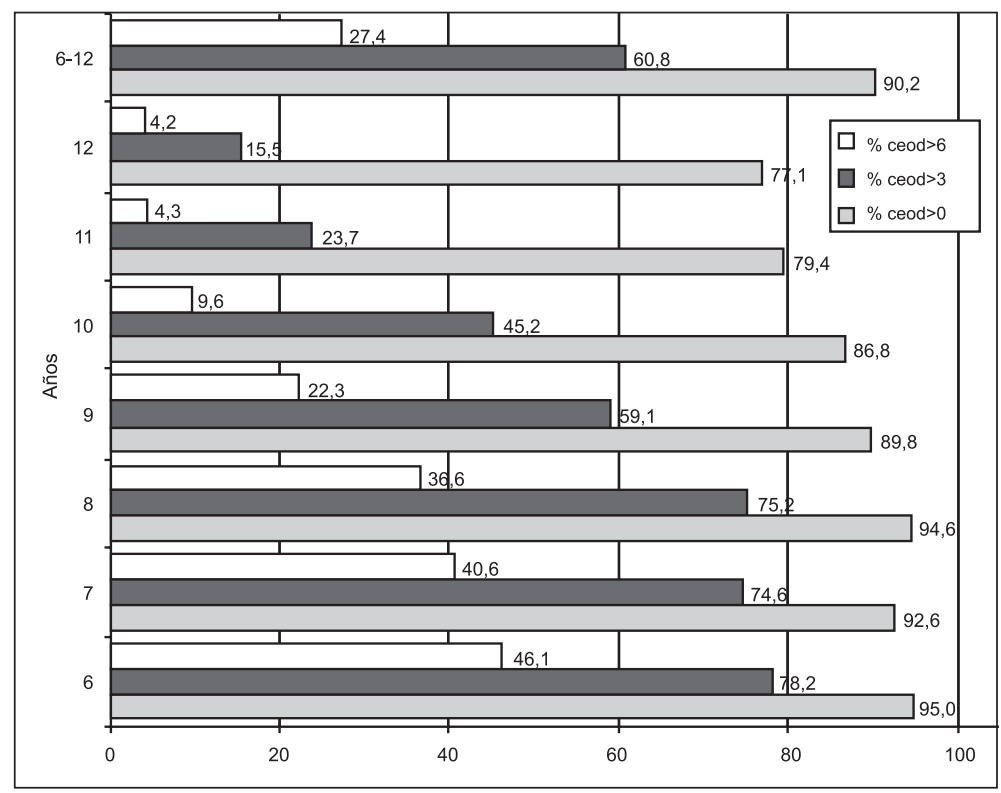

Figura 3. Distribución por edad de la prevalencia y gravedad de la caries en la dentición temporal en niños de 6 a 12 años de edad de Navolato, Sinaloa, México, 2004.

dentición temporal y permanente. La proporción de niños que no presentaba caries (libres de caries), ya fuera en su dentición temporal o permanente fue de sólo 3,7\%. La prevalencia de caries en la dentición primaria y permanente fue de 90,2 y $82 \%$, respectivamente, y en la dentición primaria para los de 6 años de edad fue de $95 \%$. En cuanto a la gravedad, $60,8 \%$ presentó ceod $\geq 3$ y $47,8 \%$ tuvo CPOD $\geq 3$. Por otra parte, en
27,4 y $9,0 \%$ se observó gravedad marcada de caries (ceod/CPOD > 6) en la dentición temporal y permanente, respectivamente. El cuadro 1 presenta la distribución del índice de caries significativa por edad. Este índice fue de 5,91 para toda la población y de 10,87 en la dentición permanente de los niños de 12 años de edad y 10,52 en la dentición temporal de los niños de 6 años. 


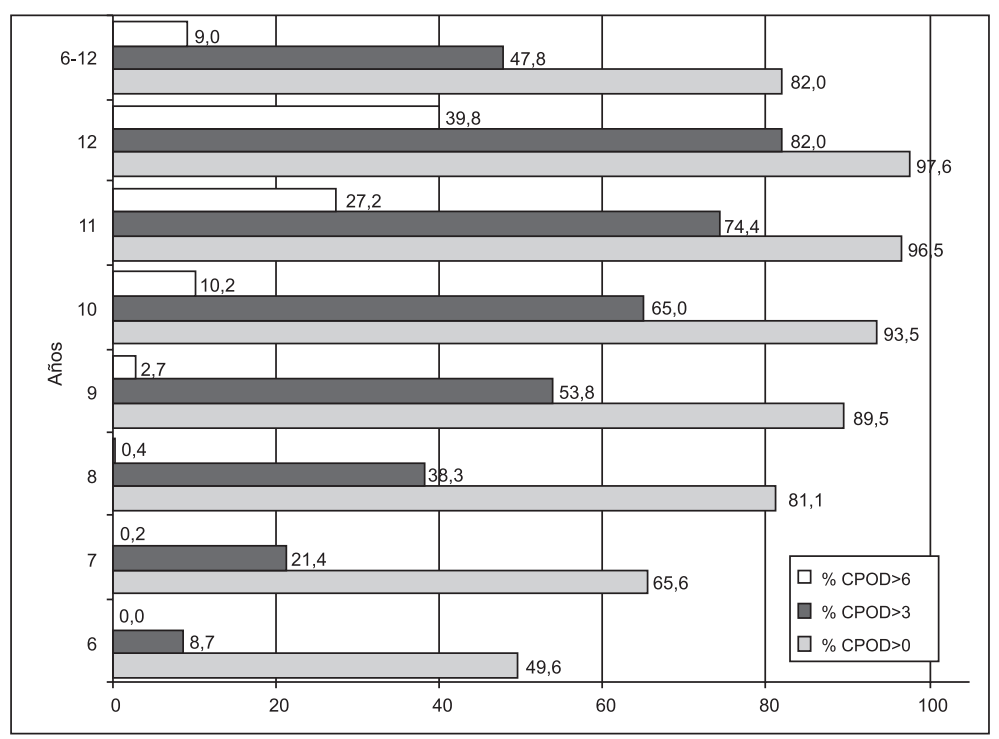

Figura 4. Distribución por edad de la prevalencia y la gravedad de la caries en la dentición permanente en niños de 6 a12 años en Navolato, Sinaloa, México, 2004.

Cuadro 1. Promedio de dientes con necesidades de restauración; porcentaje de sujetos con necesidades de tratamiento pulpar e índice de caries significativo (SiC) en niños de 6 a 12 años de edad de Navolato, Sinaloa, México, 2004.

\begin{tabular}{lccccc}
\hline Edad & $\begin{array}{c}\text { 1 superficie } \\
\text { media } \pm \text { sd (\%) }\end{array}$ & $\begin{array}{c}\geq 2 \text { superficies } \\
\text { media } \pm \text { sd (\%) }\end{array}$ & $\begin{array}{c}\text { Tx pulpar } \\
\text { media } \mathbf{~ s ~ d ~ ( \% ) ~}\end{array}$ & $\begin{array}{c}\text { SiC } \\
\text { temporal }\end{array}$ & $\begin{array}{c}\text { SiC } \\
\text { permanente }\end{array}$ \\
\hline 6 & $3,43 \pm 2,09(92,6)$ & $2,78 \pm 2,51(75,3)$ & $0,09 \pm 0,34(7,4)$ & 10,52 & 2,65 \\
7 & $3,15 \pm 2,05(91,1)$ & $3,04 \pm 2,75(76,8)$ & $0,07 \pm 0,41(5,0)$ & 9,31 & 3,68 \\
8 & $3,03 \pm 2,00(90,1)$ & $3,71 \pm 3,04(81,0)$ & $0,09 \pm 0,35(6,9)$ & 8,79 & 4,15 \\
9 & $2,82 \pm 1,89(89,9)$ & $3,72 \pm 2,94(79,8)$ & $0,06 \pm 0,28(4,3)$ & 7,36 & 4,78 \\
10 & $2,52 \pm 1,90(86,3)$ & $3,62 \pm 2,57(84,1)$ & $0,08 \pm 0,39(5,7)$ & 6,01 & 6,16 \\
11 & $2,75 \pm 2,07(87,1)$ & $3,35 \pm 2,29(86,6)$ & $0,06 \pm 0,27(4,4)$ & 4,56 & 8,89 \\
12 & $3,18 \pm 2,36(91,8)$ & $3,48 \pm 2,28(86,9)$ & $0,05 \pm 0,25(4,9)$ & 4,26 & 10,87 \\
\hline $6-12$ & $2,94 \pm 2,04(89,6)$ & $3,41 \pm 2,71(81,1)$ & $0,07 \pm 0,34(5,5)$ & 8,28 & 5,91 \\
\hline
\end{tabular}

Cuadro 2. Distribución por sexo de los índices de caries en niños de 6 a12 años de edad de Navolato, Sinaloa, México, 2004.

\begin{tabular}{|c|c|c|c|c|c|c|}
\hline & CPOD & Ceod & CPOD $>0$ & ceod $>0$ & CPOD>3 & ceod $>3$ \\
\hline \multicolumn{7}{|l|}{ Sexo } \\
\hline Hombres $(n=1.431)$ & $2,97 \pm 2,46$ & $4,75 \pm 3,25$ & $79,9 \%$ & $89,8 \%$ & $44,4 \%$ & $61,3 \%$ \\
\hline Mujeres $(n=1.587)$ & $3,49 \pm 2,91$ & $4,61 \pm 3,16$ & $83,9 \%$ & $90,5 \%$ & $50,9 \%$ & $60,2 \%$ \\
\hline
\end{tabular}

En un análisis del tipo de lesión observamos que del promedio del componente "dientes cariados" del índice ceod (cd = 3,91), 37,1\% de esas lesiones eran tipo $\mathrm{d} 1=1,45)$, las cuales representaban $35,2 \%$ a los seis años y $42,2 \%$ a los doce. En relación con la dentición permanente, las lesiones no cavitadas (d1) tuvieron un promedio de 1,96 , lo cual representó $63,4 \%$ del promedio 3,91 ) del componente "dientes cariados" (CD) en la dentición permanente.

Las niñas se vieron más afectadas que los niños por la caries en la dentición permanente, tanto en 
la experiencia como en la prevalencia y la gravedad, pero no así en la dentición temporal (cuadro 2). Se observó que conforme aumentaba la edad se incrementaba la experiencia de caries, mientras el ceod disminuía con la edad. Los momios de presentar caries en la dentición permanente $(C P O D>0)$ en los niños afectados por caries en la dentición temporal (que tuvieron ceod $>0)$ fueron casi tres veces $(\mathrm{RM}=2,93)$ cuando se comparó con los momios de los niños que no presentaron caries en la dentición temporal $($ ceod $=0)$.

Del total de niños, $89,6 \%$ necesitaba al menos la restauración de una superficie dental, mientras que $81,1 \%$ necesitaba al menos una restauración de dos o más superficies ya fuera en la dentición temporal o permanente. Respecto a las necesidades de tratamiento pulpar, $5,5 \%$ presentó esta necesidad en cualquiera de sus denticiones (cuadro 2). El índice de cuidados para el total de la muestra fue de $4,3 \%$ en la dentición permanente y de $8,3 \%$ en la temporal.

\section{Discusión}

En este estudio se observó alta prevalencia de caries, $90 \%$ en la dentición temporal y $82 \%$ en la permanente, así como alto promedio de dientes afectados por niños, 4,68 $\pm 3,21$ dientes temporales y $3,24 \pm 2,72$ dientes permanentes. Existen diversas formas de medir o detectar la presencia de caries. En este estudio se utilizó, además de lo propuesto por la OMS, las lesiones tipo 1 de Pitts, aspecto que impone limitaciones a la comparación con estudios futuros.

Tomando en cuenta las lesiones cavitadas y al comparar con estudios $(6,30)$ previos realizados en México, nuestros resultados fueron más desfavorables. Sin embargo, en ellos no se midieron de la misma forma las lesiones de caries. Igualmente, es inevitable resaltar la baja presencia de dientes restaurados $(<10 \%$ de los índices ceod/CPOD), común en países en vías de desarrollo (6,30-32), lo que de una manera u otra refleja o podría estar reflejando, por un lado, la nula importancia que tiene la salud bucal en la población, en este caso entre las madres/tutores de los niños, al igual que la observada en otras poblaciones mexicanas (33) y, por otro lado, el acceso que tienen los individuos a los servicios tanto curativos como de promoción de la salud bucal. Sin embargo, es necesario destacar que en la dentición primaria las lesiones no cavitadas representaron menos de $40 \%$ del componente "dientes cariados", pero en la dentición permanente se observaron resultados distintos; las lesiones no cavitadas fueron el mayor porcentaje de ese componente (>60\%). Esto hace necesario contar con programas que prevengan la cavitación de estas lesiones.

Por otra parte, el presente estudio confirmó lo observado en otras investigaciones $(6,30,32)$ con relación a la edad y a la aparición de caries en la dentición permanente: a mayor edad, mayor presencia de caries. Esta relación ocurrió tanto en los índices y la prevalencia de caries en cada grupo etáreo como en el SiC.

Al igual que en otros estudios similares realizados en nuestro país $(7,20)$, la prevalencia de caries fue superior en las niñas que en los niños en la dentición permanente (aún después de ajustar por edad y número de dientes presentes en boca); esta diferencia puede deberse a la erupción de este tipo de dientes, la cual ocure más temprano en las niñas que en los niños. Este dato fue corroborado al realizar las pruebas estadísticas correspondientes. No observamos diferencia estadística de la edad por sexo $(p>0,05)$. Por otro lado, cuando se realizó una prueba sobre el número de dientes presentes, las niñas tenían mayor número de dientes presentes en boca que los niños $(15,23$ vs. 13,33; $p<0,05)$ aún después de ajustar por edad (la diferencia media ajustada por edad fue de 1,63 dientes). Sin embargo, sería conveniente realizar otras investigaciones para determinar la causa de estas diferencias en la aparición de caries y no quedarnos en la simple aseveración de que a mayor número de dientes, mayor número de caries, sino tomar en cuenta los aspectos biológicos, socioeconómicos y culturales, así como la dieta y las actitudes frente a la salud bucal en uno y otro género.

El hallazgo de la asociación entre la presencia de caries en la dentición temporal y la dentición permanente se ha demostrado en estudios transversales $(6,32)$ y estudios longitudinales 
$(34,35)$, lo cual pone de manifiesto la necesidad de cuidar los dientes temporales para mejorar el ambiente bucal y preparar la erupción de los dientes permanentes.

En cuanto al método de detección de caries empleado, Burt (36) señala que los estudios transversales pueden subestimar las condiciones reales debido a las imperfecciones de los exámenes clínicos. Sin embargo, también argumenta que la habilidad en la detección de la enfermedad por parte de los examinadores nunca es perfecta. Hasta el más meticuloso método puede dar diferentes resultados en diferentes momentos del día, lo cual podría deberse a la fuente de luz, las habilidades del investigador y la agudeza visual, entre otros. Por ello, mientras más sensitivo sea el sistema de detección, más lesiones se encuentran. Así por ejemplo, en este estudio se incluyeron las lesiones de caries no cavitadas y no solo las cavitadas $(28,29)$, lo cual explica la alta experiencia y prevalencia de caries observada, aunque también es una limitación para la comparación con estudios nacionales. A diferencia de otros estudios $(37,38)$, en los que se ha observado que la mayor prevalencia de lesiones en la dentición primaria corresponde a las no cavitadas, en el nuestro se encontró que en la dentición temporal prevalecieron las lesiones cavitadas. Por otro lado, con relación a estudios $(39,40)$ realizados en dentición permanente, nuestros resultados son semejantes; las lesiones no cavitadas fueron las más prevalentes. Sin embargo, en un estudio (41) realizado en Escocia en niños de 5 a 15 años, se reportó que en la dentición temporal las lesiones cavitadas fueron las más prevalentes y en la dentición permanente las más frecuentes fueron las no cavitadas, resultados éstos iguales a los nuestros.

Un primer paso para la planeación de los servicios de salud bucal es el conocimiento del perfil epidemiológico de la población. Así, con base en el conocimiento generado a partir del presente estudio se pueden planear diferentes estrategias tanto preventivas como curativas en estos escolares. Por otra parte, la OMS ha insistido en la necesidad de realizar estudios epidemiológicos como forma de monitorizar las condiciones de salud bucal de las poblaciones. En este sentido es necesario efectuar estudios que revelen la situación de la población para llevar a cabo la planeación de programas de salud bucal que se adapten a las necesidades de tratamiento restaurador de la población. A propósito de esta última observación, es necesario mencionar que, algunas veces, como ocurrió en la población donde se realizó el estudio, no sólo es necesario prevenir sino también tratar las lesiones de caries existentes.

A manera de conclusión podemos decir que se observaron altos índices de caries, así como alta prevalencia y gravedad de caries en ambas denticiones. Este estudio encontró que en la dentición temporal prevalecieron las lesiones cavitadas, mientras que en la permanente, las lesiones no cavitadas fueron las más prevalentes. Comparando nuestros resultados con las metas de salud bucal de la OMS, podemos decir que no se lograron los objetivos de la OMS y la FDI para el año 2000, lo que confirma la necesidad de mejorar los programas preventivos ya existentes, así como diseñar programas de tratamiento de amplia cobertura y bajo costo, e incluso contemplar su universalización, lo que permitiría resolver las necesidades inmediatas de atención dental de la población escolar. Dado que las enfermedades bucales presentan un efecto acumulativo, y tomando en cuenta los resultados, es necesario implementar estrategias que mejoren las condiciones de salud bucal en estos niños y lograr las metas para el año 2015, estrategias que deben incluir, además del componente preventivo, un componente restaurativo.

\section{Conflicto de intereses}

Ninguno.

\section{Referencias}

1. Pitts NB, Stamm JW. International Consensus Workshop on Caries Clinical Trials (ICW-CCT) - Final consensus statements: Agreeing where the evidence leads. J Dent Res 2004;83:125-8.

2. Maupomé G, Borges SA, Ledesma C, Herrera R, Leyva ER, Navarro A. Prevalencia de caries en zonas rurales y periurbanas marginadas. Salud Publica Mex 1993;35:357-67.

3. Maupomé G. An introspective qualitative report on dietary patterns and elevated levels of dental decay in 
a deprived urban population in northern Mexico. ASDC J Dent Child 1998;65:276-85.

4. Irigoyen ME, Maupome G, Mejia AM. Caries experience and treatment needs in a 6- to 12-year-old urban population in relation to socio-economic status. Community Dent Health 1999;16:245-9.

5. Herrera MS, Medina-Solís CE, Rosado-Vila G, Minaya-Sánchez $M$, Vallejos-Sánchez AA, Casanova-Rosado JF. Prevalencia, severidad de caries y necesidades de tratamiento en preescolares de una comunidad suburbana de Campeche-2001. Bol Med Hosp Infant Mex 2003;60:189-96.

6. Casanova-Rosado AJ, Medina-Solís CE, CasanovaRosado JF, Vallejos-Sánchez AA, Maupomé G, Ávila-Burgos L. Dental caries and associated factor in Mexican schoolchildren aged 6-13 years. Acta Odontol Scand 2005;63:245-51.

7. Mendoza-Roaf $\mathbf{P}$, Pozos-Radillo E, Balcazar-Partida N, Valadéz-Figueroa I, Pando-Moreno M, Guerra JF. Caries dental en escolares de 6 y 12 años de edad y su relación con nivel socioeconómico y sexo en Guadalajara. Pract Odontol 1999;20:12-5

8. US Department of Health and Human Services. Oral health in America: a report of the surgeon general. Rockville: US Department of Health and Human Services, National Institutes of Dental and Craniofacial Research, National Institutes of Health; 2000.

9. Pitts NB, Stamm JW. Proceeding clinical. Preface. J Dent Res 2004;83:4-5.

10. Donahue GJ, Waddell N, Plough AL, del Aguila MA, Garland TE. The ABCDs of treating the most prevalent childhood disease. Am J Public Health 2005;95:1322-4.

11. Marthaler TM. Changes in dental caries 1953-2003. Caries Res 2004;38:173-81.

12. Holst A, Braune $\mathbf{K}$, Kjellberg $\mathbf{M}$. Changes in caries experience among 6-year-olds in Blekinge, Sweden between 1994 and 2000. Swed Dent J 2004;28:129-35.

13. Pieper K, Schulte AG. The decline in dental caries among 12-year-old children in Germany between 1994 and 2000. Community Dent Health 2004;21:199-206.

14. Carvalho JC, D'Hoore W, Van Nieuwenhuysen JP. Caries decline in the primary dentition of Belgian children over 15 years. Community Dent Oral Epidemiol 2004;32:277-82.

15. Bonecker M, Cleaton-Jones $\mathbf{P}$. Trends in dental caries in Latin American and Caribbean 5-6- and 11-13-yearold children: a systematic review. Community Dent Oral Epidemiol 2003;31:152-7.

16. de la Rosa M. Dental caries and socioeconomic status in Mexican children. J Dent Res 1978;57:453-7.

17. Escarza-Mestas ME. Morbilidad bucal en escolares del Distrito Federal. Secretaria de Salubridad y Asistencia. México DF: Dirección General de Estomatología; 1980. p.40-3.
18. Sánchez-Pérez L. Caries dental en el sur del D.F. Pract Odontol 1987;8:25-30.

19. Irigoyen ME, Lopez SA, Armendáriz DA, Baz G. Caries y necesidades de atención en una población infantil del Estado de México. Pract Odontol 1994;15:3741.

20. Moreno-Altamirano A, Carreón-García J, AlvearGalindo G, López-Moreno S, Vega-Franco L. Riesgo de caries en escolares de escuelas oficiales de la ciudad de México. Rev Mex Pediatr 2001;68,6:228-3.

21. Irigoyen ME, Sánchez-Hinojosa G. Changes in dental caries prevalence in 12-year-old students in the state of México after 9 years of salt fluoridation. Caries Res 2000;34:303-7.

22. INEGI-DGG. Superficie de la República Mexicana por Estados. 1999. [Consultado abril de 2005]. Disponible en: http://mapserver.inegi.gob.mx/geografia/espanol/ estados/sin ubic_geo.cfm?c=4 42\&e $=25 \&$ CFID $=$ $384248 \&$ CFTOKEN $=73540955$

23. INEGI. Sinaloa. XII Censo General de Población y Vivienda 2000. Resultados Preliminares. [Consultado abril de 2005]. Disponible en: http:// mapserver.inegi.gob.mx/geografia/espanol/estados/ MapDivMppal.cfm? $I d E d o=25 \&$ sist $=0$.

24. Bratthall D. Introducing the significant caries index together with a proposal for a new global oral health goal for 12-year-olds. Int Dent J 2000;50:378-84.

25. Walsh J. International patterns of oral health care - the example of New Zealand. N Z Dental J 1970;66:14352.

26. Jong A. Dental public health community dentistry. St. Louis: Mosby Co; 1981. p.74-88.

27. WHO. Oral Health Survey - Basics Methods. $4^{\text {th }}$ ed. Geneva: World Health Organization; 1997.

28. Pitts NB, Longbottom C. Preventive Care Advised (PCA)/Operative Care Advised (OCA) - Categorising caries by the management option. Community Dent Oral Epidemiol 1995;23:55-9.

29. Pitts NB. Clinical diagnosis of dental caries: a European perspective. J Dent Educ 2001;65:972-8.

30. Juárez-López ML, Hernández-Guerrero JC, Jiménez-Farfán $\mathbf{D}$, Ledesma-Montes $\mathbf{C}$. Prevalencia de fluorosis dental y caries en escolares de la ciudad de México. Gac Med Mex 2003;139:221-5.

31. Mello TR, Antunes JL. Prevalence of dental caries in schoolchildren in the rural area of Itapetininga, Sao Paulo State, Brazil. Cad Saude Publica 2004;20:829-35.

32. Herrera M, Medina-Solís CE, Maupomé G. Experiencia y prevalencia de caries dental en escolares de León Nicaragua. Gac Sanit 2005;19:302-6.

33. Maupomé G. An introspective qualitative report on dietary patterns and elevated levels of dental decay in 
a deprived urban population in northern Mexico. ASDC J Dent Child 1998;65:276-85.

34. Peretz B, Ram D, Azo E, Efrat Y. Preschool caries as an indicator of future caries: a longitudinal study. Pediatr Dent 2003;25:114-8.

35. Li Y, Wang W. Predicting caries in permanent teeth from caries in primary teeth: an eight-year cohort study. J Dent Res 2002;81:561-6.

36. Burt AB. How useful are cross-sectional data from surveys of dental caries? Community Dent Oral Epidemiol 1997;25:36-41.

37. Warren JJ, Levy SM, Kanellis MJ. Dental caries in the primary dentition: assessing prevalence of cavitated and noncavitated lesions. J Public Health Dent 2002:62:109-14.
38. Autio-Gold JT, Tomar SL. Prevalence of noncavitated and cavitated carious lesions in 5-year-old head start schoolchildren in Alachua County, Florida. Pediatr Dent 2005;27:54-60.

39. Skold UM, Klock B, Rasmusson CG, Torstensson T. Is caries prevalence underestimated in today's caries examination? A study on 16-year-old children in the county of Bohuslan, Sweden. Swed Dent J 1995; 19:213-7.

40. Machiulskiene V, Nyvad B, Baelum V. Prevalence and severity of dental caries in 12-year-old children in Kaunas, Lithuania 1995. Caries Res 1998;32:175-80.

41. Rimmer PA, Pitts NB. Effects of diagnostic threshold and overlapped approximal surfaces on reported caries status. Community Dent Oral Epidemiol 1991;19:205-12.

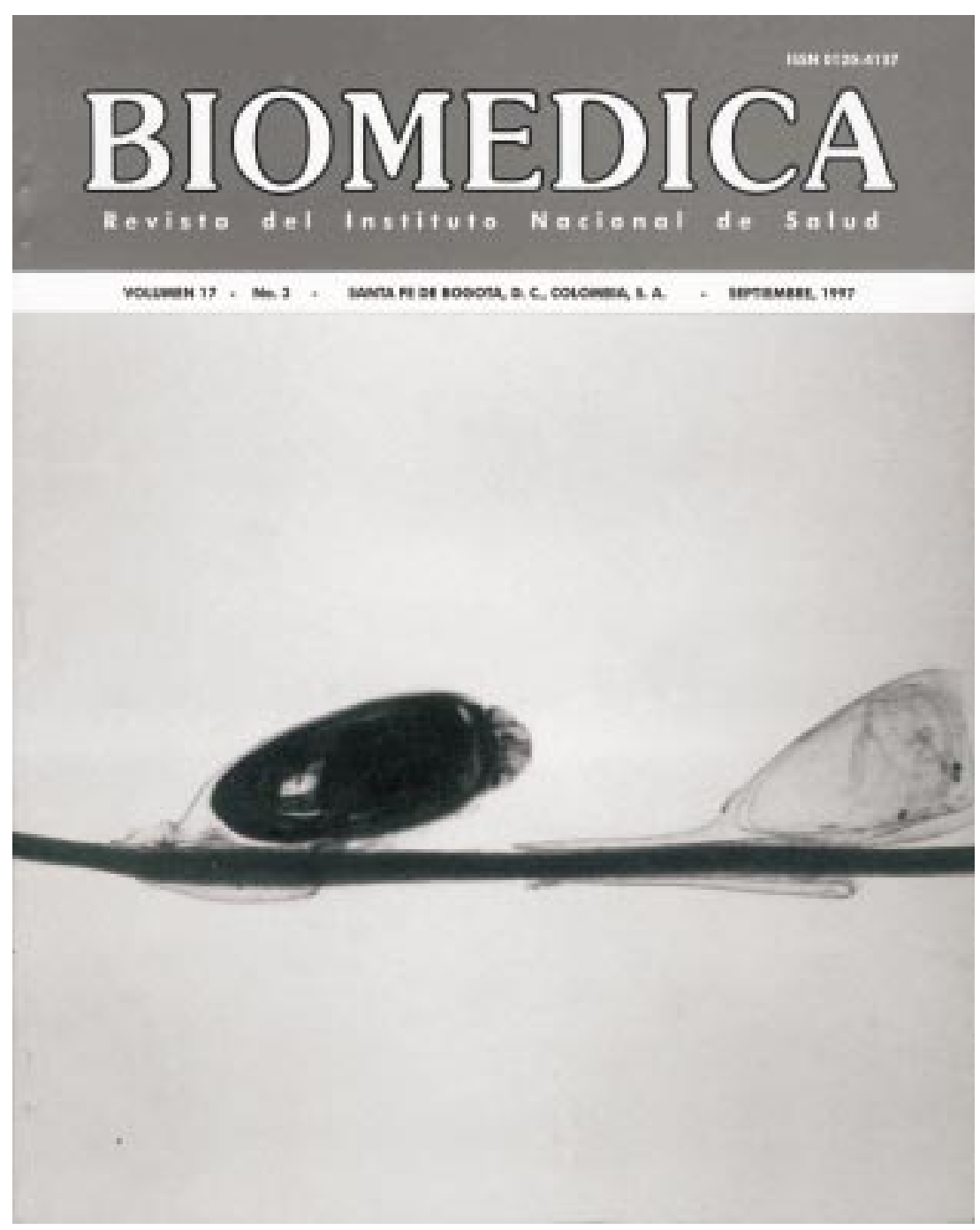

